\title{
A Dynamic Non-Singleton Fuzzy Logic System for DS/CDMA Communications
}

\author{
A. Pérez-Neira, J. Cid Sueiro, J. Roca, M. A. Lagunas \\ Dpt. of Signal Theory and Communications - Universitat Politècnica de Catalunya \\ c/ Gran Capità s/n. Campus Nord UPC. Edifici D5 - 08034 Barcelona (SPAIN) \\ Fax: +34-3-4016447 e-mail: \{anuska\}@gps.tsc.upc.es
}

\begin{abstract}
$^{1}$
We investigate a dynamic fuzzy logic system, specifically, its non-Singleton generalization, and derive a novel adaptive fuzzy line enhancer that successfully solves the non-linear problem of narrowband interference (NBI) estimation and rejection in DS/CDMA for nonstationary environments. The NBI suppression performance of this method is compared with the performance of the linear and nonlinear estimatorsubstracter NBI suppression techniques for low and high Signal to Noise Ratios (SNR's). It is seen that the proposed method outperforms all the previous nonlinear methods of NBI suppression whatever the SNR is, while almost attains the good performance of the linear ALE for low SNR and slow varying interference.
\end{abstract}

\section{Introduction}

Code-Division multiple-access (CDMA) implemented with direct-sequence spread-spectrum DS-SS signaling is among the most promising multiplexing technologies for cellular telecommunications services, such as personal communications, mobile telephony, and indoor wireless networks. One of the chief advantages of the spreadspectrum communication system is that the wide-band signals can share bandwidth with narrow-band communication systems without undue degradation of either system's performance. Although spread-spectrum communication is inherently resistant to the narrow-band interference (NBI) caused by coexistence with conventional communications, substantial performance gains can be achieved through the use of active NBI suppression prior to despreading and demodulating. Over the past two decades, a significant body of research has been concerned with the development of techniques for active NBI suppression in spread-spectrum systems. Early work in this area focused on techniques based on the

\footnotetext{
"This work was partially supported by the National Plan of Spain, CICYT, TIC96-0500-C10-01.

J.Cid Sueiro is with the ETSI Telecomunicacion, The Spanish University of Valladolid.
}

linear signal processing regimes of adaptive linear transversal filtering and Fourier-domain filtering (see [1] for a review). More recently, model-based techniques that employ non-linear filtering have been used to enhance the NBI mitigation capabilities in CDMA systems (see [2-4]). However, non-linear techniques present a greater computational burden than linear filters. Additionally, they cannot be guaranteed to converge to the globally optimum solution. Finally, non-linear NBI suppressors are very sensitive to the noise level.

In this work we design an adaptive fuzzy NBI suppressor or adaptive line enhancer (ALE) which overcomes the problems mentioned above, specially for very narrow interference. The proposed method outperforms all the previous nonlinear methods of NBI suppression whatever the SNR is. Additionally, the fast acquisition time that the fuzzy ALE presents makes it highly suitable for non-stationary communication channels.

\section{Background and signal model}

The received baseband digital direct-sequence signal can be modeled as

$$
z(t)=s(t)+n(t)+i(t)
$$

where $i(t)$ and $n(t)$ represent the narrow-band interference signal and the ambient channel noise, respectively and the DS-SS signal $s(t)$ is given by

$$
s(t)=\sum_{k=-M}^{M} b_{k} m(t-k T)
$$

where $2 \mathrm{M}+1$ is the number of data symbols per frame, $\mathrm{T}$ is the symbol interval, and where $\left\{b_{k}\right\}$ and $\mathrm{m}(\mathrm{t})$ denote, respectively, the symbol stream and normalized signaling waveform

$$
m(t)=\sum_{k=0}^{N_{c}-1} c_{k} q\left(t-k T_{c}\right)
$$

where $\mathrm{Nc}$ is the number of PN chips per message bit, $\mathrm{T}_{c}$ is the chip interval such that $\mathrm{T}=\mathrm{N}_{\mathrm{c}} \mathrm{T}_{\mathrm{c}}$, $\mathrm{c}_{\mathrm{k}}$ is the kth chip of the $P N$ sequence and $q(t)$ is a rectangular pulse of duration $T_{c}$.

It is assumed that $m(t)$ is supported only on the interval $[0, T]$ and has unit energy, and that $\left\{b_{k}\right\}$ is a 
collection of independent equiprobable random \pm 1 variables.

Suppose that the received signal is chip-matched and sampled at the chip rate of the PN sequence. We thus have

$$
z_{k}=s_{k}+n_{k}+i_{k}
$$

where $\left\{s_{k}\right\},\left\{n_{k}\right\}$ and $\left\{i_{k}\right\}$ are assumed to be mutually independent. We have assumed that $n(t)$ is bandlimited and becomes white after sampling. For the interference, we have considered that its bandwidth is small compared with $1 / \mathrm{Tc}$. Finally, since the PN sequence is random, we can assume $\left\{s_{k}\right\}$, to be a sequence of i.i.d. random variables taking values of \pm 1 with equal probability.

The estimator-substracter NBI suppression methods (also called Adaptive Line Enhancers or ALE's) are based on the following idea. Since the spread-spectrum signal has a nearly flat spectrum, it cannot be predicted accurately from its past value without explicit use of knowledge of the spreading code. On the other hand, the interfering signal, being narrow-band, can be predicted accurately. These methods essentially form a replica of the NBI which can be substracted from the received signal to enhance the wide-band components. The linear estimator-substracter methods have primarily involved the use of linear transversal prediction or interpolation filters to create the NBI replica. Such a filter forms a linear prediction of the received signal based on a fixed number of previous samples (9), or a linear interpolation based on a fixed number of past and future samples. This estimate is substracted from the appropriately timed received signal to obtain the error signal to be used as input to the SS user signature sequence correlator.

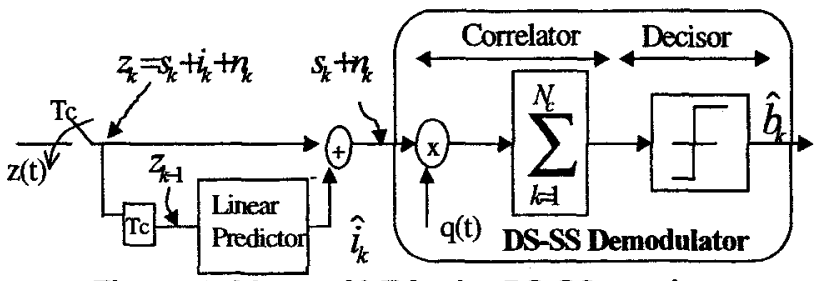

Figure 1. Linear ALE in the DS-SS receiver

For narrow-band interference added to a spreadspectrum signal in an AWGN environment, the prediction of the interferer takes place in the presence of both Gaussian and non-Gaussian noise. The non-Gaussian noise is the spread-spectrum signal itself. In such a nonGaussian environment, linear methods are no longer optimal, and nonlinear techniques offer improved suppression capability over linear methods [2-4]. Essentially, the nonlinear filters provide decision feedback that suppresses the spread-spectrum signal form the observations. When the decision feedback is an accurate estimate of the SS signal (i.e. low ambiental noise power), the filter adaptation is done in essentially Gaussian noise, i.e., observations from which the spreadspectrum signal has been removed.

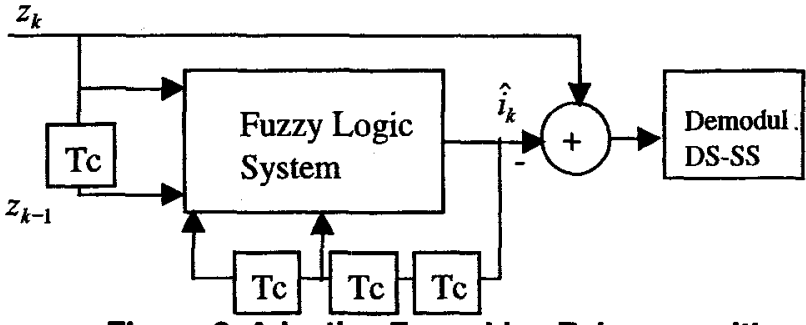

Figure 2. Adaptive Fuzzy Line Enhancer with memory.

This work develops a new non-linear ALE. As fig.2 depicts, the fuzzy system defines a nonlinear "black box" interference estimator. No Decision Directed learning is necessary, thus making the proposed system more robust in front of noise and with less computational complexity. As the output of the system at instant $k$ depends on some of the past input and output values, the system is said to be a dynamic system with memory.

\section{Dynamic fuzzy logic filter with memory}

The estimate of the interference $i_{k}$ based on observations until time $k$ is referred to as the filtering problem. It is known that the optimum of such estimate, in the minimum mean-square error sense, is the conditional expectation $\hat{i}_{k}=E\left\{i_{k} / \mathbf{z}_{k}\right\}$ (where $\mathbf{z}_{\mathbf{k}}$ is a vector containing all the observations up to time $k$ ). When the observations and the interference are Gaussian process, the optimal estimates can be recursively obtained from the well-known Kalman filter. However, as commented before our estimation problem is nonGaussian.

The fuzzy logic system that we propose to estimate $i_{k}$ can be viewed as a series expansions of fuzzy basis functions $(\mathrm{FBF}) g_{j}(\mathbf{x})[5]$

$$
y=f(\mathbf{x})=\sum_{j=1}^{M} g_{j}(\mathbf{x}) \theta_{j}
$$

where $\sigma_{j} \in R$ are constants. Our goal is to approximate $\mathrm{y}=\hat{i}_{k}$ from the input vector $\mathrm{x}=\left[\hat{i}_{k-3} \hat{i}_{k-2} z_{k} z_{k-1}\right]$. The FBF in (5) are algebraic superpositions of fuzzy membership functions. The membership functions to combine at each $g_{j}(\mathbf{x})(j=1 . . M)$ depend on the rule $j$. In this work, the introduction of the two feedback outputs in the rules models the dynamics of the non-stationary channel in the same way as the process equation in the Kalman filter does. Additionally, the additive fuzzy system that we present is a model free universal approximator [5-9], which computes a conditional mean $\mathrm{E}[\mathrm{Y} / \mathrm{X}=\mathrm{x}]$ and thus compute a least mean-square nonlinear estimate of the random variable $Y$ based on the knowledge of the random vector $X$ [7].

In this paper, we consider a fuzzy system whose basic configuration consists of four principal elements: 
fuzzifier, fuzzy rule base, fuzzy inference engine, and defuzzifier. Within each component, there are many different choices that can be made and many combinations of these choices result in different fuzzy logic systems or FLS. Next, the design of the proposed FLS is described.

\subsection{Fuzzy sets and fuzzification}

As fig. 2 has shown the fuzzy system $\mathrm{F}$ defines the map $f: U \subseteq R^{4} \rightarrow R$. In order to reduce the dimension of the rule base we compute the differences between $z_{k-1}$ and the other variables by taking $z_{k-I}$ as a reference. This gives the reduced fuzzy system $f: U \subseteq R^{3} \rightarrow R$ with $\mathrm{M}$ rules of the form IF $\left(i_{k-3}-z_{k-1}\right.$ is $\left.A_{1}{ }^{j}\right)$ AND $\left(i_{k-2}-z_{k-1}\right.$ is $A_{2}{ }^{j}$ ) AND $\left(z_{k}-z_{k-1}\right.$ is $\left.A_{3}{ }^{j}\right)$ THEN $\left(i_{k}-z_{k-1}\right.$ is $\left.B^{j}\right)$, where $(j$ $=1 . . \mathrm{M})$ and $\mathrm{A}($.$) and \mathrm{B}($.$) stand for the input term set and$ the output term set respectively.

Fig.4 shows the overlapping regions or fuzzy sets $\operatorname{Ai}(.) \in R$ designed in this work. Each fuzzy set contains an object $x$ to degree ai $(x), a \mathrm{ai}(\mathrm{x}): \mathrm{U}->[0,1]$ is the membership function. As explained later in this section, the fuzzy system uses these sets to classify or quantize the measured inputs. We note that due to the noise, these inputs are vague and, therefore, the fuzzy sets conform in a natural situation when describing the possible values of the 3 measurements in $\mathbf{x}$.

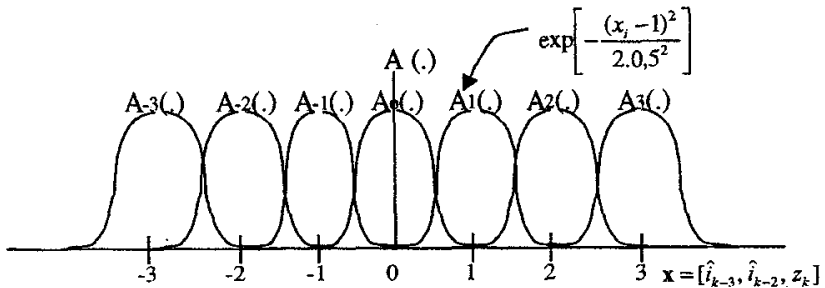

Figure 4. Fuzzy term set for the input variable

There are different methods to determine a fuzzy membership function. It is worth noting that a membership function may be subjective, but not arbitrary. Since in this work, we assume statistical inputs, the membership function estimation based on the input data probability density functions (pdf) shall be appropriate [10]. In this way, we relate the fuzzy membership functions to physical properties of the system. From eq.(4) we know the conditional probability density (pdf) function of $i_{\mathrm{k}-1}, p\left(i_{k-1} / z_{k-1}\right)$. Therefore, if we relate the fuzzy sets $A_{ \pm 1}$ with this pdf, we can say that whenever the input falls inside these fuzzy sets, this input will be related to some degree with $i_{k-1}$. This dynamic designed fuzzy sets (dynamic because their position depend on the value of $z_{k-1}$ ) act as a reference for locating values $i_{k-2}, i_{k-3}$ in a slow varying narrowband signal. Additionally, to obtain the fuzzy set for $z_{\mathrm{k}}$ we note that $p\left(z_{k} / i_{k-1}\right)$ can be assumed to be two displaced Gaussians. Thus, the fuzzification of $z_{\mathrm{k}}$ is done by means of the same fuzzy set term $\mathrm{A}($.$) as the one depicted in fig.4.$

Finally, the output fuzzy sets $B($.), which quantized in a fuzzy way the possible values of the estimated interference values $\hat{i}_{k}$, have been designed as $M$ Gaussian functions of unity area and of equal variance. $M$ is the number of IF-THEN rules. Their means are initially the same as those in fig.4, however, they can be modified by a LMS type algorithm as we comment later in this section.

We note that in order to save computation the input fuzzy sets of fig. 4 and the output fuzzy sets can be designed as symmetric triangles with a width greater than the Gaussian noise variance.

Once the input fuzzy sets are designed, the fuzzifier maps a crisp measurement of the input variable $\mathbf{x}=\mathrm{x}_{0} \in U$ into a fuzzy set on $U$. There are two main types of fuzzification: 1) The singleton fuzzifier maps the crisp measurement $\mathbf{x}_{0}$ into a fuzzy set $\mathrm{V}$ whose membersip function $v(x)$ is a delta pulse $v(x)=\delta\left(x-x_{o}\right)$.2) The nonsingleton fuzzifier performs a mapping from the observed crisp input space $\mathbf{x}_{\mathbf{o}}$ into a fuzzy set $\mathbf{V}$ with support $\mathbf{x}$ and membership function $v(\mathbf{x})$, where $v(\mathbf{x})$ achieves maximum value at $\mathbf{x}=\mathbf{x}_{0}$ and decreases while moving away from $\mathbf{x}_{0}$. We assume that fuzzy set $\mathrm{V}$ is normalized so that $\mathrm{v}\left(\mathrm{x}_{0}\right)=1$ and $\mathrm{v}(\mathrm{x})=\mathrm{v}\left(\mathrm{x}_{1}\right) \cdot \mathrm{v}\left(\mathrm{x}_{2}\right) \cdot \mathrm{v}\left(\mathrm{x}_{3}\right)$, with $x_{1}=\hat{i}_{k-3}, x_{2}=\hat{i}_{k-2}, x_{3}=z_{k}$. Examples of such membership functions are: the Gaussian $v\left(x_{i}\right)=\exp \left\{-\left(x_{i o}-x_{i}\right)^{2} / 2 c^{2}\right\}$ or the triangular $v\left(x_{i}\right)=\max \left\{0,1-\mid\left(x_{i o}-x_{i}\right) / c\right\}$, where $\mathrm{x}_{\mathrm{io}}$ and $\mathrm{c}$ are, respectively, the mean and spread of the fuzzy sets. Note that nonsingleton fuzzification is especially useful in cases where the available training data, or the input data to the fuzzy logic system, are corrupted by noise. The larger the values of the spread of the above membership functions imply that more noise is anticipated to exist in the given data.

\subsection{Fuzzy Rule Base}

The fuzzy system to design just relies on the slow varying nature of the NB interference in order to model its behavior by means of linguistic IF-THEN rules. These IFTHEN rules are the core of the fuzzy system to design. The interference range is quantized in regions or fuzzy sets $\mathrm{Bi}($.$) and, from a reference point, the evolution of the$ interference among this regions is followed by means of $M$ linguistic IF-THEN rules of the type

$$
R_{m}: I F \hat{i}_{k-3} \text { is } A_{1}^{m} \text { and } \hat{i}_{k-2} \text { is } A_{2}^{m} \text { and } z_{k} \text { is } A_{3}^{m} \text { THEN } \hat{i}_{k} \text { is } B^{m}
$$

where $\mathrm{m}=1 \ldots \mathrm{M}$ and $A_{j}^{m}(\mathrm{j}=1,2,3)$ is any of the input fuzzy subsets plotted in fig. 4 . The predictor of interference $i_{k}$ is constructed based on the $M$ rules. Each rule $R_{m}$ can be viewed as a fuzzy implication which is a fuzzy set $R_{m}($. 
in UxR with membership function $r_{m}(\mathbf{x}, y)=a_{1}^{m}\left(x_{1}\right) * a_{2}^{m}\left(x_{2}\right) * a_{3}^{m}\left(x_{3}\right) * b^{m}(y)$, where the most commonly used operations for "*" in engineering are "product" and "min". In this work we have used the "product" operation.

The fuzzy rules can be sistematically derived by considering all the possible combinations among the 7 membership functions $\left(7^{3}=343\right)$. However, in this work, this rule explotion is dramatically reduced to less than $\mathbf{7 5}$ rules by avoiding those that, for slow varying interferences, are impossible or not frequent such as:

$$
\text { IF } \hat{i}_{k-3} \text { is } A_{-3} \text { and } \hat{i}_{k-2} \text { is } A_{3} \text { and } z_{k} \text { is } A_{-3} \text { THEN } \hat{i}_{k} \text { is } B \text { ? }
$$

or controversial such as:

$$
\text { IF } \hat{i}_{k-3} \text { is } A_{0} \text { and } \hat{i}_{k-2} \text { is } A_{0} \text { and } z_{k} \text { is } A_{o} \text { THEN } \hat{i}_{k} \text { is } B_{?}\left\{\begin{array}{l}
B_{1} \\
B_{-1}
\end{array}\right.
$$

Another important point is the easy initialization of the consequents of the rule base or FAM's (Fuzzy Associative Memories). Just some expert reasoning that takes into account the slowly varying nature of the interference is needed. An example is the following rule:

$$
\text { IF } \hat{i}_{k-3} \text { is } A_{-1} \text { and } \hat{i}_{k-2} \text { is } A_{0} \text { and } z_{k} \text { is } A_{1} \text { THEN } \hat{i}_{k} \text { is } B_{2}
$$

\subsection{Fuzzy inference engine}

The fuzzy inference engine is decision making logic which employs fuzzy rules from the fuzzy rule base to determine a mapping from the fuzzy sets in the input space $U$ to the fuzzy sets in the output space $R$. Let $V$ be a fuzzy set in $U$; then each $R_{m}$ determines a fuzzy set $V_{o} R_{m}$ in $\mathrm{R}$ based on the sup-star compositional rule of inference [5]: membership $\left\{V_{0} R_{m}\right\}(y)=\sup _{\mathbf{x} \in U}\left[v(\mathbf{x}) * r_{m}(\mathbf{x}, y)\right]$

In the case of singleton fuzzification $v(\mathbf{x})=\delta\left(\mathbf{x}-\mathbf{x}_{i}\right)$ and results in

$$
m e m b .\left\{V o R_{m}\right\}(y)=a_{1}^{m}\left(\hat{i}_{k-3}\right) \cdot a_{2}^{m}\left(\hat{i}_{k-2}\right) \cdot a_{3}^{m}\left(\hat{z}_{k}\right) \cdot b^{m}(y)=w^{m}(\mathbf{x}) \cdot b^{m}(y)
$$

where $w^{m}$ is the firing strength or weight of the mth rule. In summary, all the M rules of the FAM's are activated parallely and imply a fixed number of sums and multiplications. At instant of time $k$, the result of the inference of each rule can be expressed as a matrix multiplication .

In the case of non-singleton fuzzification the firing strengths are $w^{m}=\prod_{n=1}^{3} v\left(x_{n, \text { sup }}\right) \cdot a_{1}^{m}\left(x_{n, \text { sup }}\right)$ where $\mathrm{x}_{\mathrm{n}, \text { sup }}$ is the suprema of $\left(v\left(x_{n}\right), a_{1}^{m}\left(x_{n}\right)\right)$. Under a signal and systems perspective the inference engine is a bank of filters in the $U$ domain whose outputs are sampled at their maximum output value. The impulse response of each filter is $a^{m}(\mathbf{x})$ and the input is $\mathbf{v}(\mathbf{x})$.

Finally, we use the arithmetic addition (i.e. engineering connective) as a means of aggregation instead of a t-norm.

$$
B=\sum_{m=1}^{M} w^{m} B^{m}
$$

\subsection{Defuzzifier}

After the fuzzy inference, the defuzzifier performs a mapping from the fuzzy sets in R to crisp points in R. We apply the centroid or center of mass defuzzifier in order to approximate a conditional mean estimate. For symmetric output fuzzy sets $B^{m}$ of unit area the centroid of the designed additive fuzzy system can be computed as a convex sum of the $m$ then-part set centroids

$$
\hat{i}_{k}=\frac{\int y B(y) d y}{\int B(y) d y}=\frac{\sum_{m=1}^{M} \bar{y}_{m} \cdot w^{m}(\mathbf{x})}{\sum_{m=1}^{M} w^{m}(\mathbf{x})}=\sum_{m=1}^{M} g_{m}(\mathbf{x}) \theta_{m} ;
$$

where $\bar{y}_{m}=$ centroid $\left\{B_{m}(y)\right\}$ and $\theta_{m}=\bar{y}_{m}$

Note that we have finally come to the functional expression (5), where the FBF $\mathrm{g}_{\mathrm{m}}(\mathrm{x})$ are convex coefficients or discrete probability weights.

\section{Fuzzy filters with learning}

The resulting fuzzy system of eq. (7) can uniformely approximate any continuous (or bounded measurable) function $f$ on a compact domain [7-9], in order to reduce the approximation error we propose to adjust some of the singleton and non-singleton system parameters by a learning process.

In order to perform the learning we settle for stochastic gradient descent on the squared error doing so through iterated use of the chain rule of differential calculus

$$
\gamma(k+1)=\gamma(k)-\mu \frac{\partial e(k)}{\partial f(k)} \frac{\partial f(k)}{\gamma(k)}
$$

being $\gamma$ any of the parameters to be adjusted. We define the error at instant $k, e(k)$, as desired performance minus actual performance. However, in our case we want to emphasize that the desired performance does not come from a training sequence but from feedback decision of the system (i.e., Decision Directed learning). That is, we resort to a non-supervised learning algorithm and apply it to each fuzzy system output $\hat{i}_{k}$ in order to minimize

$$
e(k)=\left|\left(z_{k}-\hat{i}_{k}\right)-h\left(z_{k}-\hat{i}_{k}\right)\right|^{2}
$$

where function $h($.$) is either h()=.\operatorname{sign}($.$) or$ $h()=.\tanh ($.$) for low or high noise power scenarios,$ respectively. This approximate conditional mean nonlinearities track the SS signal well if there is no noise.

The dependence of the designed dynamic fuzzy system output at time $k$ on the values of the outputs at time $k-I$ makes this learning task more complicated than that of training static systems. Therefore, in order to obtain recursions suitable for real-time training, we 
consider that the parameter to adjust change from iteration to iteration. This implies that the real-time algorithm will not follow the exact negative gradient of the total error surface. The difference, however usually becomes very small as the learning rate $\mu$ becomes small.

The centroid $\theta_{\mathrm{m}}$ in eq.(7) lies the simplest learning law. To simplify it more we adapt the same non-linear LMS strategy as in [2-4] and implement

$\sigma_{m}(k+1)=\sigma_{m}(k)+\mu e(k) g_{m}(\mathbf{x}(k)) \quad m=1 . M$

that is $\frac{\partial e(k)}{\partial f(k)}$ of eq. (8) is not computed.

The singleton fuzzy filter plus the non-linear LMS learning of eq.(10) is called in this work the predictive fuzzy filter. We also propose an alternative that refines the estimate of $\hat{i}_{k}$ by computing $\bar{i}_{k}$

$$
\bar{i}_{k}=\sum_{m=1}^{M} \theta_{m}(k+1) g_{m}(\mathbf{x}(k))
$$

after the learning iteration of eq.(10) and before processing a new sample data $\mathbf{x}_{k+1}$. This alternative is called estimative fuzzy filter.

When the noise power is not negligible, the fuzzy filter should include the right amount of input uncertainty. Thus, the non-singleton version should be taken into account. In this case, the best results, balancing computational complexity and performance, have been obtained just training the width of the input fuzzy set $\mathrm{V}$. In this case, assuming Gaussian input fuzzy sets, to apply the update equation in eq.(8), the following derivatives in (12) and (13) have to be computed

$$
\frac{\partial e(k)}{\partial f(k)}=\sqrt{e(k)}\left[1-\frac{1}{p_{k}} \sec h^{2}\left(\frac{z_{k}-\hat{i}_{k}}{p_{k}}\right)\right]
$$

where $p_{k}$ is the prediction error variance plus the AWGN ambiental noise variance (see. [4] for more details)

$$
\frac{\partial f(k)}{\partial \gamma(k)}=\frac{1}{\sum_{m=1}^{M} w^{m}(k)}\left[\sum_{m=1}^{M} \theta_{m} w^{m}(k) r_{m}(k)-f(k) \sum_{m=1}^{M} w^{m}(k) r_{m}(k)\right]
$$

where $w^{m}$ was defined in section 3 and

$$
\gamma(k)=c_{x}^{2}(k) ; \quad r_{m}(k)=\sum_{i=1}^{3} \frac{\left(x_{I o}(k)-m_{A_{i}^{m}}\right)^{k}}{\left(c_{x}^{2}+c_{A_{i}^{m}}^{2}\right)^{2}}
$$

being: $m_{A_{t}^{m}}$ the mean of the Gaussian antecedent fuzzy set $A_{l}^{m}$ in the $\mathrm{m}_{\mathrm{th}}$ rule, and, $\mathrm{c}_{\mathrm{x}}$ and $\mathrm{c}_{\mathrm{A}}$, respectively, the variance of the Gaussian input and antecedent fuzzy sets.

\section{Simulations}

In this section we report on simulations carried out to evaluate the performance of the proposed algorithms. In order to compare the NBI suppression capabilities of the different techniques described above, we consider that the interference is modeled as consisting of either a number of continuous wave tones of equal amplitud and with random phase or a filtered narrow-band Gaussian noise process (i.e., a second-order AR signal with both poles at 0.99). Using these signals and a DS/SS signature sequence of length 10 , the simulations can be devided in two groups. 1) The first group are low noise simulations and employ the singleton fuzzy ALE: the noise power is held constant at $\sigma^{2}=-20 \mathrm{~dB}$ below the SS signal power (after despreading), while the interference power is varied from 20 to $5 \mathrm{~dB}$ relative to a unity $\mathrm{SS}$ power signal, i.e. SIR from -20 to $-5 \mathrm{~dB}$. 2) The second group are high noise simulations and employ both singleton and nonsingleton fuzzy ALE's: the SIR is held constant at $-20 \mathrm{~dB}$ and the noise power is increased from $-20 \mathrm{~dB}$ to $0 \mathrm{~dB}$ relative to the SS signal after despreading.

In the first group of simulations our performance measure is the commonly used Signal to Noise Ratio (SNR) improvement defined in [2] as a measure of interference rejection. The SNR improvement is the ratio of SNR at the output of filtering to the SNR at the input, which reduces to

$$
S N R \text { improvement }=10 \log \left(\frac{E\left(z_{k}-\left.s_{k}\right|^{2}\right)}{E\left(\left|\left(z_{k}-\hat{i}_{k}\right)-s_{k}\right|^{2}\right)}\right)
$$

We note that in this measurement the noise includes interference $i_{k}$ and AWGN channel noise $n_{k}$. All results are obtained based on 20 trials and, for each trial, 3000 data points (i.e., chips) are computed. Table I summarizes the results and compares them with the predictorinterpolating linear filter (i.e. TS-LMS: Two Sided Least Mean Square filter [4]) and with the so-called DR2D [4]. The singleton fuzzy ALE is used without and with Decision Directed LMS (i.e., fuzzy+LMS). In both cases, the acquisition time was almost zero. In the fuzzy+LMS case, in order to carry out a fair comparison, the so-called predictive algorithm of section 4 has been considered. Gaussian membership functions are employed. However, if triangular membership functions are used instead to lessen the computational burden, the performance just degrades in 1 or $2 \mathrm{~dB}$ 's. Also, in the case of AR interference no difference exists if the 72 rules are reduced to 32 . From the obtained results we note the following:

- For the AR case, the fuzzy ALE not only outperforms the techniques proposed in [2-4] but also equals the upper bound with the predictive algorithm. To stress the effectiveness against the narrowband interferer, the table gives an upper bound on the SNR improvement for the $A R$ case assuming that the narrowband interference is predicted with noiseless accuracy, $\sigma^{2}=0$.

- For the single tone sinusoidal interference the performance is not so good as in [4] for very low SIR. 
This fact is due to the quickly speed of change of the value of the interfering signal. If we wish better results, we have to assign more membership functions to the inputs to cover the variations of the interferer. When the so-called estimative algorithm is used, however, the results improve due to the noise filtering capability of the fuzzy system. The SNR improvement is then 35.3, $34.1,32.6$ and $28.3 \mathrm{~dB}$ for SIR of $-20,-15,-10$ and -5 respectively.

In contrast to the existing non-linear ALE's, which just operate for high SNR $\left(\sigma^{2}<-20 \mathrm{~dB}\right)$, in the second group of simulations higher noise levels are taken into account. The performance measure is now the Bit Error Rate or BER, which is plotted against the SNR without any interference, i.e., the signal-to-white Gaussian noise ratio $\mathrm{Eb} /$ No. In this way, the results can be compared with those in [1].

All results are obtained based on 10 trials and, for each trial, 10.000 data points (i.e. chips) are computed. Fig. 5 compares the results of the non-singleton fuzzy ALE with the singleton one. The SIR is $-20 \mathrm{~dB}$ and the interference are 100 tones occupying $10 \%$ of the Band. The non-singleton filter improves the results of the singleton one. We note that the better results are obtained for the non-singleton with input membership functions of width twice the noise deviation. This fact confirms the design rule that the membership functions should be equal to or greater than the pdf (consistency principle [11]). As a reference the figure also shows the results obtained with the Linear Predictor (LP) of 4 taps and the LP with the Matched Filter for a $20 \%$ band interference.

Finally, to show the convergence of the trained nonsingleton fuzzy ALE, fig. 6 shows the input fuzzy membership function widths for the so-called nonsupervised training (13) $(\mu=0.01)$ and for a supervised training that would know the interference. We note that the training converges, for different initializations, to the AWGN noise deviation $(\sigma)$ although $2 \sigma$ gives better BER results as fig. 5 shows.

\begin{tabular}{|c|c|c|c|c|}
\hline \hline Input SNR $(\mathrm{dB})$ & -20 & -15 & -10 & -5 \\
\hline \multicolumn{5}{|c|}{ AR interference (32 rules) } \\
\hline Upper bound & 40 & 35 & 30 & 25 \\
\hline TS-LMS/DR2D [4] & $26.9 / 37$ & $22 / 32.6$ & $18 / 28.1$ & $13 / 23$ \\
\hline Fuzzy & 35.7 & 31.6 & 26.8 & 21.9 \\
\hline Fuzzy +LMS( $\mu=1)$ & 39.4 & 35.1 & 30.4 & 25.6 \\
\hline Sinusoidal interference (1 tone), w $_{\text {nor }}=\mathbf{0 . 1 5}(\mathbf{7 2}$ rules) \\
\hline TS-LMS/DR2D [4] & $28 / 38.5$ & $23 / 33.6$ & $18 / 28.7$ & $13 / 24$ \\
\hline \multicolumn{6}{|c|}{ Fuzzy } & 22.7 & 26.8 & 23.3 & 20.2 \\
\hline Fuzzy +LMS & 29.5 & 30.1 & 28.8 & 24.3 \\
\hline
\end{tabular}

Table 1. Comparative SNR improvement

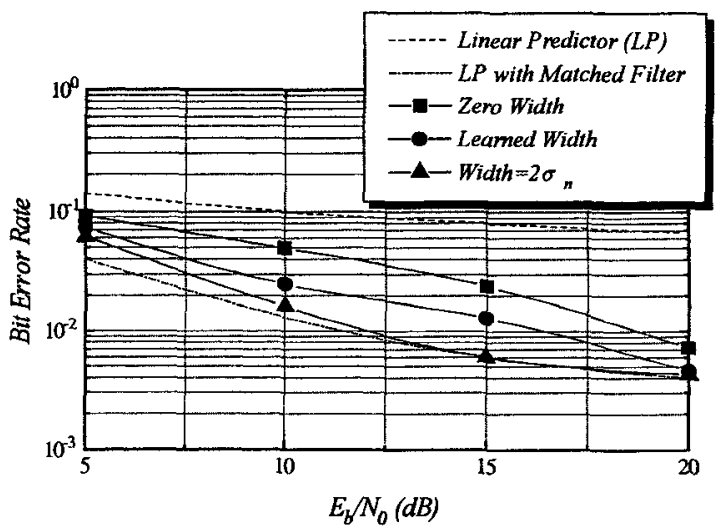

Figure 5. BER for $\mathrm{SIR}=-20 \mathrm{~dB}$

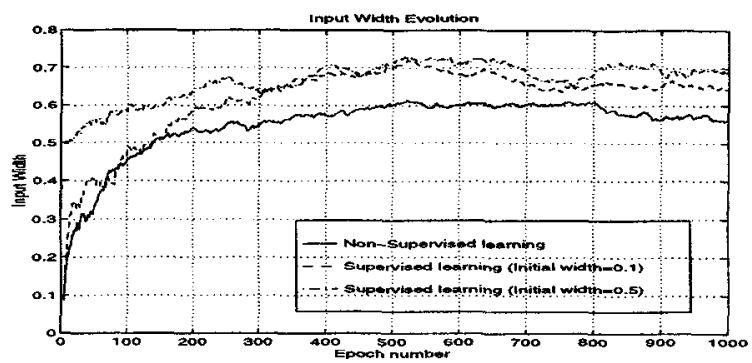

Figure 6. Width learning for $\sigma=0.7, \mathrm{SIR}=-20 \mathrm{~dB}$

\section{References}

[1] J.Ketchum, J.Proakis, "Adaptive algorithms for estimating and suppressing narrowband interference in PN SS systems," IEEE Trans. Comm. Vol. 30, May 1982.

[2] R.Vijayan, H.V.Poor, "Nonlinear techniques for interference suppression in SS systems," IEEE Trans. on Comm. Vol. 38, July 90.

[3] L. Rush, V.Poor, "Narrowband interference suppression in CDMA SS comm.," IEEE Trans. On comm., vol. 42, Feb.March/April 1994.

[4] W.Wu, F.Yu, "New nonlinear algorithms for estimating and suppressing narrowband interference in DS-SS systems," IEEE Trans. on Comm. Vol. 44, April 1996.

[5] L.Wang, J.M.Mendel, "Fuzzy Basis Functions, Universal Approximation, and Orthogonal Least-Squares Learning," IEEE Trans. on Neural Networks, vol. 3, no.5, Sept. 1992.

[6]B.Kosko, "Fuzzy systems as universal approximators," IEEE Trans. Comput., vol. 43, pp. 1329-1333, nov. 1994.

[7] B.Kosko, Fuzzy Engineering, Prentice-Hall, 1997.

[8] J.L.Castro, "Fuzzy Logic Controllers are universal approximators," IEEE Trans. on Syst., Man, Cyber., vol. 25 , no.4, April 1995.

[9] G.C.Mouzouris, J.M.Mendel, "Nonsingleton Fuzzy Logic Systems: Theory and Application," IEEE Trans. on Fuzzy Syst., vol.5, no.1, February 1997.

[10] R.P.Singh, W.H.Bailey, "Fuzzy Logic Applications to multisensor-multitarget correlation," IEEE Trans. on Aerospace and electronic Syst., vol. 33, no.3, July 1997.

[11] L.A.Zadeh, "Fuzzy sets as a basis for a theory of possibility," Fuzzy Sets and Systems, 1 (1978), 3-28. 